\title{
TO A QUESTION ON THE SUPERCOMPACTNESS OF ULTRAFILTER SPACES ${ }^{1}$
}

\author{
Alexander G. Chentsov \\ Krasovskii Institute of Mathematics and Mechanics, \\ Ural Branch of the Russian Academy of Sciences, \\ 16 S. Kovalevskaya str., Ekaterinburg, Russia, 620990 \\ chentsov@imm.uran.ru
}

\begin{abstract}
The space of ultrafilters of a $\pi$-system endowed with the topology of Wallman type is considered. The question on the supercompactness of this space is investigated. For this, the enveloping space of maximal linked systems with the corresponding topology of Wallman type is used. Necessary and sufficient conditions for the coincidence of the set of all ultrafilters of the initial $\pi$-system and the set of all maximal linked systems for this $\pi$-system are obtained. Specific variants of wide sense measurable spaces with this coincidence property are given.
\end{abstract}

Keywords: Maximal linked system, Topology, Supercompactness, Ultrafilter.

\section{Introduction}

In the present paper, we study ultrafilters of wide sense measurable spaces. Every such measurable space is defined by introducing a $\pi$-system of subsets of a nonempty set. More precisely, a $\pi$-system is a family of sets closed with respect to finite intersections; see [1, p. 14]. We consider only $\pi$-systems with "zero" and "unit," i.e., the empty set and the enveloping set are elements of our $\pi$-systems. This approach enables the precise description of the ultrafilter set for a given measurable space (in this connection, see $[2,3]$ ). The ultrafilter set can be equipped with a Wallman type topology. We consider the equipping of this type as a basic thing. In addition, we use a Stone type topology. As a result, a bitopological space is realized. This bitopological space can be considered as a kind of a subspace of the bitopological space of maximal linked systems with Wallman and Stone equippings.

Note that Wallman and Stone type topologies are used in the theory of extension of topological spaces (see [4; 5, Sect. 3.6] and others). Ultrafilters play an important role in general topology. Constructions involving maximal linked systems are used in connection with the notions of topological space; in addition, usually, maximal linked systems of the family of closed sets in a $T_{1}$-space are considered (see [4, Sect. 5.11], [6-8], and [9, Ch. VII, Sect. 4]). We emphasize the important result of $[8]$ on the supercompactness of metrizable compact sets.

In [10], for superextension constructions, a natural bitopological equipping was realized. In the author's consequent papers [11-14], this approach of [10] was extended to the case when a $\pi$-system is used as an anticipating measurable structure (the approach of [6-8] corresponds to the case when this $\pi$-system is the lattice of closed sets in a $T_{1}$-space). Thus, in the above-mentioned general case of an arbitrary $\pi$-system, bitopological spaces of ultrafilters and maximal linked systems were constructed (see [11-14]). In addition, in the case of maximal linked systems, a topology of Wallman type generates a supercompact space.

\footnotetext{
${ }^{1}$ This work was supported by Russian Foundation for Basic Research (project no.18-01-00410).
} 
Recall that ultrafilters are maximal linked systems. But often there are maximal linked systems that are not ultrafilters. The simplest example of such maximal linked system was given in [9, 4.18]. This example plays an important role in questions of representation of maximal linked systems.

At the same time, for some (widely understood) measurable space, all maximal linked systems are ultrafilters; in this connection, see $[15,16]$. In the present paper, we investigate this case in more details. It can be interesting because the question on the supercompactness of the ultrafilter space with a Wallman type topology arises. In addition, the supercompactness property is not, generally speaking, hereditary (see [4, Sect. 5.11]). However, in the case when all maximal linked systems are ultrafilters, the required supercompactness property of the ultrafilter space can be obtained from the similar property of the space of maximal linked systems. This approach reduces to an interesting representation, but requires finding conditions for the above-mentioned coincidence of the ultrafilter set and the set of maximal linked systems. The present paper is devoted to this issue.

\section{General notions and notation}

We use the standard set-theoretic symbolism (quantifiers and connectives); $\triangleq$ stands for the equality by definition and $\varnothing$ for the empty set. In what follows, a family is a set whose elements are also sets. We accept the axiom of choice. For any objects $x$ and $y$, we denote by $\{x ; y\}$ the set containing $x$ and $y$ (as proper elements) and not containing other elements; thus, $\{x ; y\}$ is an unordered pair of the objects $x$ and $y$. If $z$ is an object, then $\{z\} \triangleq\{z ; z\}$ is a singleton containing $z$ as a proper element. For any three objects $u, v$, and $w$, we set $\{u ; v ; w\} \triangleq\{u ; v\} \cup\{w\}$. For any objects $p$ and $q,(p, q) \triangleq\{\{p\} ;\{p ; q\}\}$ is the ordered pair [17, Ch. II, Sect. 3] with the first element $p$ and the second element $q$. If $z$ is an ordered pair, then $\operatorname{pr}_{1}(z)$ and $\operatorname{pr}_{2}(z)$ are the first and the second elements of $z$, respectively.

If $X$ is a set, then $\mathcal{P}(X)$ is, by definition, the family of all subsets of $X, \mathcal{P}^{\prime}(X) \triangleq \mathcal{P}(X) \backslash\{\varnothing\}$, and $\operatorname{Fin}(X)$ is the family of all finite sets of $\mathcal{P}^{\prime}(X)$; thus, $\operatorname{Fin}(X)$ is the family of all nonempty finite subsets of $X$. Certainly, a family can be used as $X$. In this connection, we introduce the following notation $[12$, Sect. 2$]$ for a fixed nonempty family $\mathfrak{X}$ :

$$
\begin{gathered}
\{\cup\}(\mathfrak{X}) \triangleq\left\{\bigcup_{X \in \mathcal{X}} X: \mathcal{X} \in \mathcal{P}(\mathfrak{X})\right\}, \quad\{\cap\}(\mathfrak{X}) \triangleq\left\{\bigcap_{X \in \mathcal{X}} X: \mathcal{X} \in \mathcal{P}^{\prime}(\mathfrak{X})\right\}, \\
\{\cup\}_{\sharp}(\mathfrak{X}) \triangleq\left\{\bigcup_{X \in \mathcal{K}} X: \mathcal{K} \in \operatorname{Fin}(\mathfrak{X})\right\}, \quad\{\cap\}_{\sharp}(\mathfrak{X}) \triangleq\left\{\bigcap_{X \in \mathcal{K}} X: \mathcal{K} \in \operatorname{Fin}(\mathfrak{X})\right\} .
\end{gathered}
$$

If $\mathbb{M}$ is a set and $\mathcal{M} \in \mathcal{P}^{\prime}(\mathcal{P}(\mathbb{M}))$, then

$$
\mathbf{C}_{\mathbb{M}}[\mathcal{M}] \triangleq\{\mathbb{M} \backslash M: M \in \mathcal{M}\} \in \mathcal{P}^{\prime}(\mathcal{P}(\mathbb{M}))
$$

is the family dual to $\mathcal{M}$. For any set $H$ and a family $\mathcal{H} \in \mathcal{P}^{\prime}(\mathcal{P}(H))$, we have

$$
\mathcal{H}=\mathbf{C}_{H}\left[\mathbf{C}_{H}[\mathcal{H}]\right] .
$$

For any nonempty family $\mathcal{S}$ and a set $L$, define

$$
\left(\left.\mathcal{S}\right|_{L} \triangleq\{S \cap L: S \in \mathcal{S}\} \in \mathcal{P}^{\prime}(\mathcal{P}(L))\right) \&([\mathcal{S}](L) \triangleq\{S \in \mathcal{S} \mid L \subset S\} \in \mathcal{P}(\mathcal{S})) .
$$

If $\mathbb{X}$ is a nonempty set and $\mathcal{X} \in \mathcal{P}^{\prime}(\mathcal{P}(\mathbb{X}))$, then

$$
(\mathrm{COV})[\mathbb{X} \mid \mathcal{X}] \triangleq\left\{\chi \in \mathcal{P}^{\prime}(\mathcal{X}) \mid \mathbb{X}=\bigcup_{X \in \chi} X\right\} \in \mathcal{P}\left(\mathcal{P}^{\prime}(\mathcal{X})\right)
$$


is the family of all coverings of the set $\mathbb{X}$ by sets of $\mathcal{X}$; a covering $\kappa \in(\mathrm{COV})[\mathbb{X} \mid \mathcal{X}]$ is called binary if $\kappa=\left\{X_{1} ; X_{2}\right\}$, where $X_{1} \in \mathcal{X}$ and $X_{2} \in \mathcal{X}$.

Let $\mathbb{R}$ be the real line and $\mathbb{N} \triangleq\{1 ; 2 ; \ldots\} ; \mathbb{N} \in \mathcal{P}^{\prime}(\mathbb{R})$. For $n \in \mathbb{N}$, we use the notation $\overline{1, n} \triangleq$ $\{k \in \mathbb{N} \mid k \leq n\}$. For a set $H$, denote by $H^{n}$ the set of all mappings from $\overline{1, n}$ into $H$; thus, elements of $H^{n}$ are $n$-tuples $\left(h_{i}\right)_{i \in \overline{1, n}}$, where $h_{j} \in H$ for $j \in \overline{1, n}$.

Some special families. In this subsection, we fix a nonempty set $\mathbf{I}$ and consider families of $\mathcal{P}^{\prime}(\mathcal{P}(\mathbf{I})$ ), i.e., nonempty families of subsets of $\mathbf{I}$, especially, $\pi$-systems. More precisely, let

$$
\pi[\mathbf{I}] \triangleq\left\{\mathcal{I} \in \mathcal{P}^{\prime}(\mathcal{P}(\mathbf{I})) \mid(\varnothing \in \mathcal{I}) \&(\mathbf{I} \in \mathcal{I}) \&(A \cap B \in \mathcal{I} \quad \forall A \in \mathcal{I} \quad \forall B \in \mathcal{I})\right\}
$$

be the family of all $\pi$-systems of subsets of $\mathbf{I}$ with "zero" and "unit." If $\mathcal{I} \in \pi[\mathbf{I}]$, then the pair $(\mathbf{I}, \mathcal{I})$ is considered as a measurable space in the wide sense. Denote by

$$
\tilde{\pi}^{0}[\mathbf{I}] \triangleq\{\mathcal{I} \in \pi[\mathbf{I}] \mid \forall L \in \mathcal{I} \quad \forall x \in \mathbf{I} \backslash L \quad \exists \Lambda \in \mathcal{I}:(x \in \Lambda) \&(\Lambda \cap L=\varnothing)\}
$$

the family of all separable $\pi$-systems of (1.2). Finally, we introduce the family

$$
\begin{gathered}
\pi_{*}^{\sharp}[\mathbf{I}] \triangleq\left\{\mathcal{I} \in \pi[\mathbf{I}] \mid \forall \Sigma_{1} \in \mathcal{I} \quad \forall \Sigma_{2} \in \mathcal{I} \quad \forall \Sigma_{3} \in \mathcal{I}\right. \\
\left.\left(\left(\Sigma_{1} \cap \Sigma_{2} \neq \varnothing\right) \&\left(\Sigma_{2} \cap \Sigma_{3} \neq \varnothing\right) \&\left(\Sigma_{1} \cap \Sigma_{3} \neq \varnothing\right)\right) \Longrightarrow\left(\Sigma_{1} \cap \Sigma_{2} \cap \Sigma_{3} \neq \varnothing\right)\right\} .
\end{gathered}
$$

In (1.3), we have a special variant of $\pi$-systems connected with the linkedness property that plays a basic role in this paper. We introduce the family of lattices by using only subsets of $\mathbf{I}$. More precisely, let

$$
(\mathrm{LAT})_{0}[\mathbf{I}] \triangleq\{\mathfrak{L} \in \pi[\mathbf{I}] \mid A \cup B \in \mathfrak{L} \quad \forall A \in \mathfrak{L} \quad \forall B \in \mathfrak{L}\}
$$

be the family of all lattices of subsets of $\mathbf{I}$. In addition, the following two particular cases are realized:

$$
\begin{gathered}
(\operatorname{alg})[\mathbf{I}] \triangleq\{\mathcal{A} \in \pi[\mathbf{I}] \mid \mathbf{I} \backslash A \in \mathcal{A} \quad \forall A \in \mathcal{A}\}=\left\{\mathcal{A} \in(\mathrm{LAT})_{0}[\mathbf{I}] \mid \mathbf{I} \backslash A \in \mathcal{A} \quad \forall A \in \mathcal{A}\right\}, \\
(\text { top })[\mathbf{I}] \triangleq\left\{\tau \in \pi[\mathbf{I}] \mid \bigcup_{G \in \mathcal{G}} G \in \tau \quad \forall \mathcal{G} \in \mathcal{P}^{\prime}(\tau)\right\}=\left\{\tau \in(\mathrm{LAT})_{0}[\mathbf{I}] \mid \bigcup_{G \in \mathcal{G}} G \in \tau \quad \forall \mathcal{G} \in \mathcal{P}^{\prime}(\tau)\right\} .
\end{gathered}
$$

Certainly, $(\mathbf{I}, \mathcal{A})$ with $\mathcal{A} \in(\operatorname{alg})[\mathbf{I}]$ is a usual measurable space with algebra of sets. Moreover, $(\mathbf{I}, \tau)$ with $\tau \in($ top $)[\mathbf{I}]$ is a topological space. Finally,

$$
(\operatorname{clos})[\mathbf{I}] \triangleq\left\{\mathcal{F} \in(\mathrm{LAT})_{0}[\mathbf{I}] \mid \bigcap_{F \in \mathcal{F}^{\prime}} F \in \mathcal{F} \quad \forall \mathcal{F}^{\prime} \in \mathcal{P}^{\prime}(\mathcal{F})\right\}=\left\{\mathbf{C}_{\mathbf{I}}[\tau]: \tau \in(\text { top })[\mathbf{I}]\right\} .
$$

Elements of (1.4) are closed topologies in the sense of P.S. Aleksandrov [18, Ch. 4, Sect. 1]. We introduce the family of all nonempty centered subfamilies of a $\pi$-system:

$$
(\mathrm{Cen})[\mathcal{I}] \triangleq\left\{\mathcal{Z} \in \mathcal{P}^{\prime}(\mathcal{I}) \mid \bigcap_{Z \in \mathcal{K}} Z \neq \varnothing \quad \forall \mathcal{K} \in \operatorname{Fin}(\mathcal{Z})\right\} \quad \forall \mathcal{I} \in \pi[\mathbf{I}] .
$$

Now, we recall notions connected with bases and subbases of topological spaces. In addition,

$$
\begin{gathered}
(\mathrm{BAS})[\mathbf{I}] \triangleq\left\{\beta \in \mathcal{P}^{\prime}(\mathcal{P}(\mathbf{I})) \mid\left(\mathbf{I}=\bigcup_{B \in \beta} B\right) \&\left(\forall B_{1} \in \beta \quad \forall B_{2} \in \beta \quad \forall x \in B_{1} \cap B_{2}\right.\right. \\
\left.\left.\exists B_{3} \in \beta:\left(x \in B_{3}\right) \&\left(B_{3} \subset B_{1} \cap B_{2}\right)\right)\right\}, \\
(\mathrm{cl}-\mathrm{BAS})[\mathbf{I}] \triangleq\left\{\beta \in \mathcal{P}^{\prime}(\mathcal{P}(\mathbf{I})) \mid(\mathbf{I} \in \beta) \&\left(\bigcap_{B \in \beta} B=\varnothing\right)\right. \\
\left.\&\left(\forall B_{1} \in \beta \quad \forall B_{2} \in \beta \quad \forall x \in \mathbf{I} \backslash\left(B_{1} \cup B_{2}\right) \quad \exists B_{3} \in \beta:\left(B_{1} \cup B_{2} \subset B_{3}\right) \&\left(x \notin B_{3}\right)\right)\right\} .
\end{gathered}
$$


In (1.5), bases of sets open in some topology of $\mathbf{I}$ are introduced. Similarly, in (1.6), bases of sets closed in some topology of $\mathbf{I}$ are introduced. For brevity, we call bases from (1.5) and (1.6) open and closed, respectively. Certainely, the property $\{\cup\}(\beta) \in($ top $)[\mathbf{I}]$ (the property $\{\cap\}(\beta) \in($ clos) $[\mathbf{I}]$ ) is realized for $\beta \in(\mathrm{BAS})[\mathbf{I}]$ (for $\beta \in(\mathrm{cl}-\mathrm{BAS})[\mathbf{I}])$. Moreover, we consider open and closed bases of a specific topological space. More precisely, for any topology $\tau \in($ top) $[\mathbf{I}]$, let

$$
\begin{gathered}
\left((\tau-\mathrm{BAS})_{0}[\mathbf{I}] \triangleq\{\beta \in(\mathrm{BAS})[\mathbf{I}] \mid \tau=\{\cup\}(\beta)\}\right) \\
\&\left((\mathrm{cl}-\mathrm{BAS})_{0}[\mathbf{I} ; \tau] \triangleq\left\{\beta \in(\mathrm{cl}-\mathrm{BAS})[\mathbf{I}] \mid \mathbf{C}_{\mathbf{I}}[\tau]=\{\cap\}(\beta)\right\}\right)
\end{gathered}
$$

(thus, open and closed bases of the topological space $(\mathbf{I}, \tau)$ are introduced). We introduce subbases of open and closed sets in some topology on the set $\mathbf{I}$ :

$$
\begin{gathered}
\left((\mathrm{p}-\mathrm{BAS})[\mathbf{I}] \triangleq\left\{\mathbf{x} \in \mathcal{P}^{\prime}(\mathcal{P}(\mathbf{I})) \mid\{\cap\}_{\sharp}(\mathbf{x}) \in(\mathrm{BAS})[\mathbf{I}]\right\}=\left\{\mathbf{x} \in \mathcal{P}^{\prime}(\mathcal{P}(\mathbf{I})) \mid \mathbf{I}=\bigcup_{\mathbb{X} \in \mathbf{x}} \mathbb{X}\right\}\right) \\
\&\left((\mathrm{p}-\mathrm{BAS})_{\mathrm{cl}}[\mathbf{I}] \triangleq\left\{\chi \in \mathcal{P}^{\prime}(\mathcal{P}(\mathbf{I})) \mid\{\cup\}_{\sharp}(\chi) \in(\mathrm{cl}-\mathrm{BAS})[\mathbf{I}]\right\}\right) .
\end{gathered}
$$

Now, we introduce open and closed subbases of a specific topological space. More precisely, for $\tau \in($ top $)[\mathbf{I}]$, let

$$
\begin{gathered}
\left((\mathrm{p}-\mathrm{BAS})_{0}[\mathbf{I} ; \tau] \triangleq\left\{\chi \in(\mathrm{p}-\mathrm{BAS})[\mathbf{I}] \mid\{\cap\}_{\sharp}(\chi) \in(\tau-\mathrm{BAS})_{0}[\mathbf{I}]\right\}\right) \\
\&\left((\mathrm{p}-\mathrm{BAS})_{\mathrm{cl}}^{0}[\mathbf{I} ; \tau] \triangleq\left\{\chi \in(\mathrm{p}-\mathrm{BAS})_{\mathrm{cl}}[\mathbf{I}] \mid\{\cup\}_{\sharp}(\chi) \in(\mathrm{cl}-\mathrm{BAS})_{0}[\mathbf{I} ; \tau]\right\}\right) .
\end{gathered}
$$

In (1.7), the families of open and closed subbases of the topological space $(\mathbf{I}, \tau)$ are introduced. For $\tau \in($ top $)[\mathbf{I}]$ and $x \in \mathbf{I}$, we set $N_{\tau}^{0}(x) \triangleq\{G \in \tau \mid x \in G\}$ and

$$
N_{\tau}(x) \triangleq\left\{H \in \mathcal{P}(\mathbf{I}) \mid \exists G \in N_{\tau}^{0}(x): G \subset H\right\}
$$

obtaining the family of all neighborhoods of $x$ in the topological space $(\mathbf{I}, \tau)$. In addition, for $\tau \in($ top $)[\mathbf{I}]$ and $A \in \mathcal{P}(\mathbf{I})$, we define the closure of $A$ in the topological space $(\mathbf{I}, \tau)$ :

$$
\operatorname{cl}(A, \tau) \triangleq\left\{x \in \mathbf{I} \mid A \cap H \neq \varnothing \quad \forall H \in N_{\tau}(x)\right\} .
$$

In what follows, the supercompactness property plays an important role. In this connection, we set

$$
\begin{aligned}
& ((\mathbb{S C})-\text { top })[\mathbf{I}] \triangleq\left\{\tau \in(\text { top })[\mathbf{I}] \mid \exists \mathcal{S} \in(\mathbf{p}-\mathrm{BAS})_{0}[\mathbf{I} ; \tau] \quad \forall \mathcal{G} \in(\mathrm{COV})[\mathbf{I} \mid \mathcal{S}]\right. \\
& \left.\exists G_{1} \in \mathcal{G} \quad \exists G_{2} \in \mathcal{G}: \mathbf{I}=G_{1} \cup G_{2}\right\} .
\end{aligned}
$$

Then, (1.8) is the family of all topologies converting $\mathbf{I}$ to a supercompact topological space. A supercompact $T_{2}$-space is called [4] a supercompact set. Certainly, (1.8) can be defined in terms of closed subbases (see [4-9]).

\section{The family of ultrafilters and its Stone equipping}

In what follows, we fix a nonempty set $E$ and consider its $\pi$-systems. Moreover, in this section, we also fix $\mathcal{I} \in \pi[E]$. Then

$\mathbb{F}^{\star}(\mathcal{I}) \triangleq\left\{\mathcal{F} \in \mathcal{P}^{\prime}(\mathcal{I} \backslash\{\varnothing\}) \mid(A \cap B \in \mathcal{F} \forall A \in \mathcal{F} \forall B \in \mathcal{F}) \&(\forall F \in \mathcal{F} \forall I \in \mathcal{I} \quad(F \subset I) \Longrightarrow(I \in \mathcal{F}))\right\}$

is the set of all filters of the (widely understood) measurable space $(E, \mathcal{I})$. The simplest variant of a filter is given by the following obvious definition: for $x \in E$,

$$
(\mathcal{I}-\text { triv })[x] \triangleq\{I \in \mathcal{I} \mid x \in I\} \in \mathbb{F}^{\star}(\mathcal{I}) .
$$


In (2.1), we obtain the trivial filter corresponding to the point $x$. Maximal filters are called ultrafilters. Then

$$
\begin{aligned}
& \mathbb{F}_{0}^{\star}(\mathcal{I}) \triangleq\left\{\mathcal{U} \in \mathbb{F}^{\star}(\mathcal{I}) \mid \forall \mathcal{F} \in \mathbb{F}^{\star}(\mathcal{I}) \quad(\mathcal{U} \subset \mathcal{F}) \Longrightarrow(\mathcal{U}=\mathcal{F})\right\} \\
& =\left\{\mathcal{U} \in \mathbb{F}^{\star}(\mathcal{I}) \mid \forall I \in \mathcal{I}(I \cap U \neq \varnothing \quad \forall U \in \mathcal{U}) \Longrightarrow(I \in \mathcal{U})\right\} \\
& =\{\mathcal{U} \in(\text { Cen })[\mathcal{I}] \mid \forall \mathcal{V} \in(\text { Cen })[\mathcal{I}] \quad(\mathcal{U} \subset \mathcal{V}) \Longrightarrow(\mathcal{U}=\mathcal{V})\}
\end{aligned}
$$

is the set of all ultrafilters of the measurable space $(E, \mathcal{I})$. It is known (see $[19,(5.9)])$ that

$$
\left((\mathcal{I}-\operatorname{triv})[x] \in \mathbb{F}_{0}^{\star}(\mathcal{I}) \forall x \in E\right) \Longleftrightarrow\left(\mathcal{I} \in \tilde{\pi}^{0}[E]\right) .
$$

In the general case of $\mathcal{I}, \forall \mathcal{F} \in \mathbb{F}^{*}(\mathcal{I}) \exists \mathcal{U} \in \mathbb{F}_{0}^{*}(\mathcal{I}): \mathcal{F} \subset \mathcal{U}$ (this property is established by the Zorn lemma). Thus, by $(2.1)$, we obtain $\mathbb{F}_{0}^{*}(\mathcal{I}) \neq \varnothing$. Let

$$
\Phi_{\mathcal{I}}(L) \triangleq\left\{\mathcal{U} \in \mathbb{F}_{0}^{\star}(\mathcal{I}) \mid L \in \mathcal{U}\right\} \quad \forall L \in \mathcal{I} .
$$

In addition, (2.2) and (2.3) imply the following property:

$$
\Phi_{\mathcal{I}}(L) \triangleq\left\{\mathcal{U} \in \mathbb{F}_{0}^{\star}(\mathcal{I}) \mid U \cap L \neq \varnothing \quad \forall U \in \mathcal{U}\right\} \quad \forall L \in \mathcal{I} .
$$

Representation (2.4) plays an important role in the theory of ultrafilters. Note that

$$
(\mathbb{U F})[E ; \mathcal{I}] \triangleq\left\{\Phi_{\mathcal{I}}(I): I \in \mathcal{I}\right\} \in \pi\left[\mathbb{F}_{0}^{\star}(\mathcal{I})\right]
$$

In addition, $(\mathbb{U F})[E ; \mathcal{I}] \in \pi\left[\mathbb{F}_{0}^{\star}(\mathcal{I})\right]$ and, in particular, $(\mathbb{U F})[E ; \mathcal{I}] \in(\operatorname{BAS})\left[\mathbb{F}_{0}^{\star}(\mathcal{I})\right]$. Thus, we obtain the topology of Stone type:

$$
\mathbf{T}_{\mathcal{I}}^{\star}[E] \triangleq\{\cup\}((\mathbb{U F})[E ; \mathcal{I}]) \in(\text { top })\left[\mathbb{F}_{0}^{\star}(\mathcal{I})\right] .
$$

Thus, $(\mathbb{U F})[E ; \mathcal{I}] \in\left(\mathbf{T}_{\mathcal{I}}^{\star}[E]-\operatorname{BAS}\right)_{0}\left[\mathbb{F}_{0}^{\star}(\mathcal{I})\right]$. In addition, by $(2.4)$, we have the following inclusion:

$$
(\mathbb{U F})[E ; \mathcal{I}]) \subset \mathbf{T}_{\mathcal{I}}^{\star}[E] \cap \mathbf{C}_{\mathbb{F}_{0}^{\star}(\mathcal{I})}\left[\mathbf{T}_{\mathcal{I}}^{\star}[E]\right]
$$

(for $\mathcal{I} \in(\operatorname{alg})[E],(2.5)$ becomes an equality, see [20, Remark 3.3]). Note that

$$
\left(\mathbb{F}_{0}^{\star}(\mathcal{I}), \mathbf{T}_{\mathcal{I}}^{\star}[E]\right)
$$

is a zero-dimensional $T_{2}$-space (if $\mathcal{I} \in(\mathrm{alg})[E]$, then (2.6) is a zero-dimensional compact set, see [20, Sect. 3]).

For $H \in \mathcal{P}(E)$, we set

$$
\mathbb{F}_{\mathbf{C}}^{\natural}[\mathcal{I} \mid H] \triangleq\left\{\mathcal{U} \in \mathbb{F}_{0}^{\star}(\mathcal{I}) \mid \exists U \in \mathcal{U}: U \subset H\right\}
$$

By (2.4), we have

$$
\mathbb{F}_{\mathbf{C}}^{\natural}[\mathcal{I} \mid E \backslash I]=\mathbb{F}_{0}^{\star}(\mathcal{I}) \backslash \Phi_{\mathcal{I}}(I) \quad \forall I \in \mathcal{I} .
$$

Using (2.7), we obtain the following representation:

$$
\mathfrak{F}_{\mathbf{C}}^{\natural}[\mathcal{I}] \triangleq\left\{\mathbb{F}_{\mathbf{C}}^{\natural}[\mathcal{I} \mid \Lambda]: \Lambda \in \mathbf{C}_{E}[\mathcal{I}]\right\}=\mathbf{C}_{\mathbb{F}_{0}^{\star}(\mathcal{I})}[(\mathbb{U F})[E ; \mathcal{I}]] \in(\mathrm{cl}-\operatorname{BAS})_{0}\left[\mathbb{F}_{0}^{\star}(\mathcal{I}) ; \mathbf{T}_{\mathcal{I}}^{\star}[E]\right] .
$$

Thus, by (2.8), we obtain a closed base of the topological space (2.6). In this connection, it is useful to note one representation of ultrafilters connected with (2.7) and (2.8). More precisely, from (2.6) 
and (2.8), we conclude that, for every $L \in \mathcal{I}$, the set $\Phi_{\mathcal{I}}(L)$ is the intersection of all sets of the family $\left[\mathfrak{F}_{\mathbf{C}}^{\natural}[\mathcal{I}]\right]\left(\Phi_{\mathcal{I}}(L)\right)$. In addition, by [12, Proposition 3.1], for $L \in \mathcal{I}$ and $\Lambda \in \mathbf{C}_{E}[\mathcal{I}]$, we have

$$
(L \subset \Lambda) \Longleftrightarrow\left(\Phi_{\mathcal{I}}(L) \subset \mathbb{F}_{\mathbf{C}}^{\natural}[\mathcal{I} \mid \Lambda]\right)
$$

As a corollary, we obtain the following property:

$$
\left[\mathfrak{F}_{\mathbf{C}}^{\natural}[\mathcal{I}]\right]\left(\Phi_{\mathcal{I}}(L)\right)=\left\{\mathbb{F}_{\mathbf{C}}^{\natural}[\mathcal{I} \mid \Lambda]: \Lambda \in\left[\mathbf{C}_{E}[\mathcal{I}]\right](L)\right\} \quad \forall L \in \mathcal{I} .
$$

From (2.9), we extract the useful equality

$$
\mathbb{F}_{0}^{\star}(\mathcal{I})=\left\{\mathcal{U} \in \mathbb{F}^{\star}(\mathcal{I}) \mid \forall I \in \mathcal{I}(I \in \mathcal{U}) \vee\left(\exists \Lambda \in\left[\mathbf{C}_{E}[\mathcal{I}]\right](I): E \backslash \Lambda \in \mathcal{U}\right)\right\}
$$

In connection with (2.10), we emphasize the constructions from [16, Sect. 2].

\section{Ultrafilters and maximal linked systems; topologies of Wallman type}

In what follows, unless otherwise stated, we fix a $\pi$-system $\mathcal{E} \in \pi[E]$. Since $\mathfrak{F}_{\mathbf{C}}^{\natural}[\mathcal{E}] \in$ $(\mathrm{p}-\mathrm{BAS})\left[\mathbb{F}_{0}^{\star}(\mathcal{E})\right]$, we introduce the topology of Wallman type

$$
\mathbf{T}_{\mathcal{E}}^{0}\langle E\rangle \triangleq\{\cup\}\left(\{\cap\}_{\sharp}\left(\mathfrak{F}_{\mathbf{C}}^{\natural}[\mathcal{E}]\right)\right) \in(\text { top })\left[\mathbb{F}_{0}^{\star}(\mathcal{E})\right] .
$$

In addition, we obtain the topological space

$$
\left(\mathbb{F}_{0}^{\star}(\mathcal{E}), \mathbf{T}_{\mathcal{E}}^{0}\langle E\rangle\right) .
$$

Using (3.1), we also call (3.2) a topological space of Wallman type. To investigate the properties of this topological space, we consider maximal linked systems of the $\pi$-system $\mathcal{E}$. More precisely, for $\mathfrak{X} \in \mathcal{P}^{\prime}(\mathcal{P}(E))$, we set

$$
\langle\mathfrak{X}-\operatorname{link}\rangle[E] \triangleq\left\{\mathcal{X} \in \mathcal{P}^{\prime}(\mathfrak{X}) \mid X_{1} \cap X_{2} \neq \varnothing \quad \forall X_{1} \in \mathcal{X} \quad \forall X_{2} \in \mathcal{X}\right\} .
$$

Thus, (3.3) is the family of all linked subfamilies of $\mathfrak{X}$. Moreover, for $\mathfrak{Y} \in \mathcal{P}^{\prime}(\mathcal{P}(E)$ ), we consider the family of all maximal linked systems of the family $\mathfrak{Y}$ :

$$
\langle\mathfrak{Y}-\operatorname{link}\rangle_{0}[E] \triangleq\{\mathcal{Y} \in\langle\mathfrak{Y}-\operatorname{link}\rangle[E] \mid \forall \mathcal{Z} \in\langle\mathfrak{Y}-\operatorname{link}\rangle[E] \quad(\mathcal{Y} \subset \mathcal{Z}) \Longrightarrow(\mathcal{Y}=\mathcal{Z})\}
$$

By (3.4), the family

$$
\begin{gathered}
\langle\mathcal{E}-\operatorname{link}\rangle_{0}[E]=\{\mathcal{S} \in\langle\mathcal{E}-\operatorname{link}\rangle[E] \mid \forall \tilde{\mathcal{S}} \in\langle\mathcal{E}-\operatorname{link}\rangle[E] \quad(\mathcal{S} \subset \tilde{\mathcal{S}}) \Longrightarrow(\mathcal{S}=\tilde{\mathcal{S}})\} \\
=\{\mathcal{S} \in\langle\mathcal{E}-\operatorname{link}\rangle[E] \mid \forall L \in \mathcal{E} \quad(L \cap S \neq \varnothing \quad \forall S \in \mathcal{S}) \Longrightarrow(L \in \mathcal{S})\}
\end{gathered}
$$

is defined (see [12, Sect. 4]). Note that $\forall \mathcal{S} \in\langle\mathcal{E}-\operatorname{link}\rangle[E] \exists \tilde{\mathcal{S}} \in\langle\mathcal{E}-\operatorname{link}\rangle_{0}[E]: \mathcal{S} \subset \tilde{\mathcal{S}}$; see [12, (4.5)]. Moreover (see $[12,(4.5)]$ ),

$$
\forall \mathcal{S} \in\langle\mathcal{E}-\operatorname{link}\rangle_{0}[E] \quad \forall S \in \mathcal{S} \quad \forall \Sigma \in \mathcal{E}(S \subset \Sigma) \Longrightarrow(\Sigma \in \mathcal{S}) .
$$

Note the following property [12, Sect. 4]:

$$
\mathbb{F}_{0}^{\star}(\mathcal{E})=\left\{\mathcal{U} \in\langle\mathcal{E}-\operatorname{link}\rangle_{0}[E] \mid A \cap B \in \mathcal{U} \quad \forall A \in \mathcal{U} \quad \forall B \in \mathcal{U}\right\} \in \mathcal{P}^{\prime}\left(\langle\mathcal{E}-\operatorname{link}\rangle_{0}[E]\right) .
$$

For $L \in \mathcal{E}$, we set $\langle\mathcal{E}-\operatorname{link}\rangle^{0}[E \mid L] \triangleq\left\{\mathcal{S} \in\langle\mathcal{E}-\operatorname{link}\rangle_{0}[E] \mid L \in \mathcal{S}\right\}$. Moreover, for $H \in \mathcal{P}(E)$, let

$$
\langle\mathcal{E}-\operatorname{link}\rangle_{\mathrm{op}}^{0}[E \mid H] \triangleq\left\{\mathcal{S} \in\langle\mathcal{E}-\operatorname{link}\rangle_{0}[E] \mid \exists \Sigma \in \mathcal{S}: \Sigma \subset H\right\} .
$$


Now, we introduce the following families:

$$
\begin{gathered}
\hat{\mathfrak{C}}_{\mathrm{op}}^{0}[E ; \mathcal{E}] \triangleq\left\{\langle\mathcal{E}-\operatorname{link}\rangle_{\mathrm{op}}^{0}[E \mid \Lambda]: \Lambda \in \mathbf{C}_{E}[\mathcal{E}]\right\} \in \mathcal{P}^{\prime}\left(\mathcal{P}\left(\langle\mathcal{E}-\operatorname{link}\rangle_{0}[E]\right)\right), \\
\hat{\mathfrak{C}}_{0}^{\star}[E ; \mathcal{E}] \triangleq\left\{\langle\mathcal{E}-\operatorname{link}\rangle^{0}[E \mid \Sigma]: \Sigma \in \mathcal{E}\right\}=\mathbf{C}_{\langle\mathcal{E}-\text { link }\rangle_{0}[E]}\left[\hat{\mathfrak{C}}_{\mathrm{op}}^{0}[E ; \mathcal{E}]\right] .
\end{gathered}
$$

In connection with $(3.6)$, we note that $\hat{\mathfrak{C}}_{\mathrm{op}}^{0}[E ; \mathcal{E}] \in(\mathrm{p}-\mathrm{BAS})\left[\langle\mathcal{E}-\operatorname{link}\rangle_{0}[E]\right]$. As a corollary, the following topology of Wallman type is defined (see [12, Sect. 5]):

$$
\mathbb{T}_{0}\langle E \mid \mathcal{E}\rangle \triangleq\{\cup\}\left(\{\cap\}_{\sharp}\left(\hat{\mathfrak{C}}_{\mathrm{op}}^{0}[E ; \mathcal{E}]\right)\right) \in((\mathbb{S C})-\text { top })\left[\langle\mathcal{E}-\operatorname{link}\rangle_{0}[E]\right] .
$$

In addition,

$$
\left(\langle\mathcal{E}-\operatorname{link}\rangle_{0}[E], \mathbb{T}_{0}\langle E \mid \mathcal{E}\rangle\right)
$$

is a supercompact $T_{1}$-space for which (see (3.5))

$$
\mathbf{T}_{\mathcal{E}}^{0}\langle E\rangle=\left.\mathbb{T}_{0}\langle E \mid \mathcal{E}\rangle\right|_{\mathbb{F}_{0}^{\star}(\mathcal{E})} .
$$

Thus, (3.2) is a subspace of the topological space (3.7) and

$$
\hat{\mathfrak{C}}_{\mathrm{op}}^{0}[E ; \mathcal{E}] \in(\mathrm{p}-\mathrm{BAS})_{0}\left[\langle\mathcal{E}-\operatorname{link}\rangle_{0}[E] ; \mathbb{T}_{0}\langle E \mid \mathcal{E}\rangle\right] .
$$

Now, we recall the useful corollary of the supercompactness of the topological space (3.7) connected with (3.9); more precisely, $\forall \mathcal{G} \in \mathcal{P}^{\prime}\left(\mathbf{C}_{E}[\mathcal{E}]\right)$

$$
\begin{gathered}
\left(\langle\mathcal{E}-\operatorname{link}\rangle_{0}[E]=\bigcup_{G \in \mathcal{G}}\langle\mathcal{E}-\operatorname{link}\rangle_{\mathrm{op}}^{0}[E \mid G]\right) \Longrightarrow\left(\exists G_{1} \in \mathcal{G} \quad \exists G_{2} \in \mathcal{G}:\right. \\
\left.\langle\mathcal{E}-\operatorname{link}\rangle_{0}[E]=\langle\mathcal{E}-\operatorname{link}\rangle_{\mathrm{op}}^{0}\left[E \mid G_{1}\right] \cup\langle\mathcal{E}-\operatorname{link}\rangle_{\mathrm{op}}^{0}\left[E \mid G_{2}\right]\right) .
\end{gathered}
$$

More detailed information on the topological spaces (3.2) and (3.7) can be found in $[12,15,16]$.

\section{Ultrafilters and maximal linked systems; topologies of Stone type}

We recall that, as a variant of (2.6), the zero-dimensional $T_{2}$-space

$$
\left(\mathbb{F}_{0}^{\star}(\mathcal{E}), \mathbf{T}_{\mathcal{E}}^{\star}[E]\right)
$$

is defined. We consider (4.1) as a topological space of Stone type. Now, let us recall an analog of (4.1) for the space of maximal linked systems. To this end, note that

$$
\hat{\mathfrak{C}}_{0}^{\star}[E ; \mathcal{E}] \in(\mathrm{p}-\mathrm{BAS})\left[\langle\mathcal{E}-\operatorname{link}\rangle_{0}[E]\right] .
$$

Therefore, the following topology is defined:

$$
\mathbb{T}_{\star}\langle E \mid \mathcal{E}\rangle \triangleq\{\cup\}\left(\{\cap\}_{\sharp}\left(\hat{\mathfrak{C}}_{0}^{\star}[E ; \mathcal{E}]\right)\right) \in(\text { top })\left[\langle\mathcal{E}-\operatorname{link}\rangle_{0}[E]\right] .
$$

In addition, topology $(4.2)$ converts $\langle\mathcal{E}-\operatorname{link}\rangle_{0}[E]$ to the zero-dimensional $T_{2}$-space

$$
\left(\langle\mathcal{E}-\operatorname{link}\rangle_{0}[E], \mathbb{T}_{\star}\langle E \mid \mathcal{E}\rangle\right) .
$$

Note that, by [12, Proposition 6.5], we have

$$
\mathbf{T}_{\mathcal{E}}^{\star}[E]=\left.\mathbb{T}_{\star}\langle E \mid \mathcal{E}\rangle\right|_{\mathbb{F}_{0}^{\star}(\mathcal{E})} .
$$


Thus, in view of (4.4), we conclude that the topological space

$$
\left(\mathbb{F}_{0}^{\star}(\mathcal{E}), \mathbf{T}_{\mathcal{E}}^{\star}[E]\right)
$$

is a subspace of the topological space (4.3). Moreover, by [12, Proposition 7.1], we have the inclusion

$$
\mathbb{T}_{0}\langle E \mid \mathcal{E}\rangle \subset \mathbb{T}_{\star}\langle E \mid \mathcal{E}\rangle .
$$

If $X$ is a nonempty set, $\tau_{1} \in($ top $)[X], \tau_{2} \in($ top $)[X]$, and $\tau_{1} \subset \tau_{2}$, then we call the triplet $\left(X, \tau_{1}, \tau_{2}\right)$ a bitopological space. Then $\left(\langle\mathcal{E}-\operatorname{link}\rangle_{0}[E], \mathbb{T}_{0}\langle E \mid \mathcal{E}\rangle, \mathbb{T}_{\star}\langle E \mid \mathcal{E}\rangle\right)$ and $\left(\mathbb{F}_{0}^{\star}(\mathcal{E}), \mathbf{T}_{\mathcal{E}}^{0}\langle E\rangle, \mathbf{T}_{\mathcal{E}}^{\star}[E]\right)$ are two bitopological spaces. General properties of these two bitopological spaces are given in $[12,13,16]$.

Now, let us recall some statements from [16, Sect. 7]. More precisely, to the end of this section, we suppose that $\mathcal{E} \in \tilde{\pi}^{0}[E]$. Therefore (see Sect. 2), $(\mathcal{E}-\operatorname{triv})[x] \in \mathbb{F}_{0}^{\star}(\mathcal{E}) \forall x \in E$. As a result, we obtain the mapping

$$
(\mathcal{E}-\text { triv })[\cdot] \triangleq((\mathcal{E}-\operatorname{triv})[x])_{x \in E}
$$

acting from $E$ to $\mathbb{F}_{0}^{\star}(\mathcal{E})$ and the corresponding sets-images

$$
(\mathcal{E}-\operatorname{triv})[\cdot]^{1}(A)=\{(\mathcal{E}-\text { triv })[x]: x \in A\} \in \mathcal{P}\left(\mathbb{F}_{0}^{\star}(\mathcal{E})\right)
$$

for $A \in \mathcal{P}(E)$. We can consider the closure of every such set both in the topology $\mathbf{T}_{\mathcal{E}}^{0}\langle E\rangle$ and in the topology $\mathbf{T}_{\mathcal{E}}^{\star}[E]$. In addition (see $[16,(7.2)]$ ),

$$
\operatorname{cl}\left((\mathcal{E}-\operatorname{triv})[\cdot]^{1}(A), \mathbf{T}_{\mathcal{E}}^{\star}[E]\right)=\left\{\mathcal{U} \in \mathbb{F}_{0}^{\star}(\mathcal{E}) \mid A \cap U \neq \varnothing \quad \forall U \in \mathcal{U}\right\} \quad \forall A \in \mathcal{P}(E) .
$$

Moreover, by [16, Proposition 7.1], we obtain

$$
\Phi_{\mathcal{E}}(\Sigma)=\operatorname{cl}\left((\mathcal{E}-\operatorname{triv})[\cdot]^{1}(\Sigma), \mathbf{T}_{\mathcal{E}}^{0}\langle E\rangle\right)=\operatorname{cl}\left((\mathcal{E}-\operatorname{triv})[\cdot]^{1}(\Sigma), \mathbf{T}_{\mathcal{E}}^{\star}[E]\right) \quad \forall \Sigma \in \mathcal{E} .
$$

From (4.5), we obtain the corresponding density property:

$$
\mathbb{F}_{0}^{\star}(\mathcal{E})=\operatorname{cl}\left((\mathcal{E}-\operatorname{triv})[\cdot]^{1}(E), \mathbf{T}_{\mathcal{E}}^{0}\langle E\rangle\right)=\operatorname{cl}\left((\mathcal{E}-\operatorname{triv})[\cdot]^{1}(E), \mathbf{T}_{\mathcal{E}}^{\star}[E]\right) .
$$

Relations (4.5) and (4.6) imply the following fact: in the general case $\mathcal{E} \in \tilde{\pi}^{0}[E]$, the properties of the topologies $\mathbf{T}_{\mathcal{E}}^{0}\langle E\rangle$ and $\mathbf{T}_{\mathcal{E}}^{\star}[E]$ are similar. In [16, Sects. 5, 6], sufficient conditions for the coincidence of these topologies and conditions under which $\mathbf{T}_{\mathcal{E}}^{0}\langle E\rangle \neq \mathbf{T}_{\mathcal{E}}^{\star}[E]$ are given as well as similar conditions for the topologies $\mathbb{T}_{0}\langle E \mid \mathcal{E}\rangle$ and $\mathbb{T}_{\star}\langle E \mid \mathcal{E}\rangle$.

\section{Proper maximal linked systems}

Let us emphasize some properties of the set $\langle\mathcal{E}-\operatorname{link}\rangle_{0}[E] \backslash \mathbb{F}_{0}^{\star}(\mathcal{E})$. We call elements of this set proper maximal linked systems. Let us observe some properties of such maximal linked systems (more details can be found in $[15,16]$ ). We first note the following statement from [16]:

$$
\langle\mathcal{E}-\operatorname{link}\rangle_{0}[E] \backslash \mathbb{F}_{0}^{\star}(\mathcal{E})=\left\{\mathcal{S} \in\langle\mathcal{E}-\operatorname{link}\rangle_{0}[E] \mid \exists S_{1} \in \mathcal{S} \exists S_{2} \in \mathcal{S} \exists S_{3} \in \mathcal{S}: S_{1} \cap S_{2} \cap S_{3}=\varnothing\right\} .
$$

Hence, $\forall \mathcal{M} \in\langle\mathcal{E}-\operatorname{link}\rangle_{0}[E]$,

$$
(A \cap B \cap C \neq \varnothing \quad \forall A \in \mathcal{M} \quad \forall B \in \mathcal{M} \quad \forall C \in \mathcal{M}) \Longrightarrow\left(\mathcal{M} \in \mathbb{F}_{0}^{\star}(\mathcal{E})\right) .
$$

Moreover, (4.2) and (5.1) imply the following property:

$$
\langle\mathcal{E}-\operatorname{link}\rangle_{0}[E] \backslash \mathbb{F}_{0}^{\star}(\mathcal{E}) \in \mathbb{T}_{\star}\langle E \mid \mathcal{E}\rangle .
$$

Finally, using (5.1), we obtain the following equivalence:

$$
\begin{gathered}
\left(\langle\mathcal{E}-\text { link }\rangle_{0}[E] \backslash \mathbb{F}_{0}^{\star}(\mathcal{E}) \neq \varnothing\right) \Longleftrightarrow\left(\exists \Sigma_{1} \in \mathcal{E} \quad \exists \Sigma_{2} \in \mathcal{E} \quad \exists \Sigma_{3} \in \mathcal{E}:\right. \\
\left.\left(\Sigma_{1} \cap \Sigma_{2} \neq \varnothing\right) \&\left(\Sigma_{2} \cap \Sigma_{3} \neq \varnothing\right) \&\left(\Sigma_{1} \cap \Sigma_{3} \neq \varnothing\right) \&\left(\Sigma_{1} \cap \Sigma_{2} \cap \Sigma_{3}=\varnothing\right)\right) .
\end{gathered}
$$


Theorem 1. The following equivalence holds:

$$
\left(\mathcal{E} \in \pi_{\star}^{\sharp}[E]\right) \Longleftrightarrow\left(\langle\mathcal{E}-\operatorname{link}\rangle_{0}[E]=\mathbb{F}_{0}^{\star}(\mathcal{E})\right) .
$$

P r o o f. Let $\mathcal{E} \in \pi_{\star}^{\sharp}[E]$. Consider an arbitrary $\mathcal{M} \in\langle\mathcal{E}-\operatorname{link}\rangle_{0}[E]$. Let

$$
\left(M_{1} \in \mathcal{M}\right) \&\left(M_{2} \in \mathcal{M}\right) \&\left(M_{3} \in \mathcal{M}\right)
$$

Then, by (1.3), we obtain the following implication:

$$
\left(\left(M_{1} \cap M_{2} \neq \varnothing\right) \&\left(M_{2} \cap M_{3} \neq \varnothing\right) \&\left(M_{1} \cap M_{3} \neq \varnothing\right)\right) \Longrightarrow\left(M_{1} \cap M_{2} \cap M_{3} \neq \varnothing\right) .
$$

Now, we use (5.2). More precisely, by the choice of $\mathcal{M}$, we have the following property (see (3.3)):

$$
\left(M_{1} \cap M_{2} \neq \varnothing\right) \&\left(M_{2} \cap M_{3} \neq \varnothing\right) \&\left(M_{1} \cap M_{3} \neq \varnothing\right) .
$$

From (5.6), we conclude that $M_{1} \cap M_{2} \cap M_{3} \neq \varnothing$. Since the choice of (5.5) was arbitrary, we have the property

$$
A \cap B \cap C \neq \varnothing \quad \forall A \in \mathcal{M} \quad \forall B \in \mathcal{M} \quad \forall C \in \mathcal{M} .
$$

From (5.2), the inclusion $\mathcal{M} \in \mathbb{F}_{0}^{\star}(\mathcal{E})$ follows. Thus,

$$
\langle\mathcal{E}-\operatorname{link}\rangle_{0}[E] \subset \mathbb{F}_{0}^{\star}(\mathcal{E}) .
$$

Using (3.5) and (5.7), we obtain the equality $\langle\mathcal{E}-\operatorname{link}\rangle_{0}[E]=\mathbb{F}_{0}^{\star}(\mathcal{E})$. Thus, we have the implication

$$
\left(\mathcal{E} \in \pi_{\star}^{\sharp}[E]\right) \Longrightarrow\left(\langle\mathcal{E}-\operatorname{link}\rangle_{0}[E]=\mathbb{F}_{0}^{\star}(\mathcal{E})\right) .
$$

Let $\langle\mathcal{E}-\operatorname{link}\rangle_{0}[E]=\mathbb{F}_{0}^{\star}(\mathcal{E})$. Let us prove that $\mathcal{E} \in \pi_{\star}^{\sharp}[E]$. On the contrary, let $\mathcal{E} \notin \pi_{\star}^{\sharp}[E]$. From (1.3), we obtain for some $\Sigma_{1} \in \mathcal{E}, \Sigma_{2} \in \mathcal{E}$, and $\Sigma_{3} \in \mathcal{E}$

$$
\left(\Sigma_{1} \cap \Sigma_{2} \neq \varnothing\right) \&\left(\Sigma_{2} \cap \Sigma_{3} \neq \varnothing\right) \&\left(\Sigma_{1} \cap \Sigma_{3} \neq \varnothing\right) \&\left(\Sigma_{1} \cap \Sigma_{2} \cap \Sigma_{2}=\varnothing\right) .
$$

Then, by $(5.3),\langle\mathcal{E}-\operatorname{link}\rangle_{0}[E] \backslash \mathbb{F}_{0}^{\star}(\mathcal{E}) \neq \varnothing$, a contradiction. Thus, the required inclusion $\mathcal{E} \in \pi_{\star}^{\sharp}[E]$ holds. Therefore, we have the implication

$$
\left(\langle\mathcal{E}-\operatorname{link}\rangle_{0}[E]=\mathbb{F}_{0}^{\star}(\mathcal{E})\right) \Longrightarrow\left(\mathcal{E} \in \pi_{\star}^{\sharp}[E]\right) .
$$

Using (5.8), we obtain (5.4).

Remark 1. Theorem 1 implies the following equality:

$$
\pi_{\star}^{\sharp}[E]=\left\{\mathcal{L} \in \pi[E] \mid\langle\mathcal{L}-\operatorname{link}\rangle_{0}[E]=\mathbb{F}_{0}^{\star}(\mathcal{L})\right\} .
$$

\section{Supercompact spaces of ultrafilters}

Consider some corollaries of Theorem 1. The $\pi$-systems from (1.3) play an important role in these constructions. The following statement is an obvious corollary of (3.8) and Theorem 1.

Proposition 1. If $\mathcal{E} \in \pi_{\star}^{\sharp}[E]$, then $\mathbf{T}_{\mathcal{E}}^{0}\langle E\rangle \in((\mathbb{S C})-$ top $)\left[\mathbb{F}_{0}^{\star}(\mathcal{E})\right]$. 
Proposition 1 motivates special investigation of specific variants of $\pi$-systems from $\pi_{\star}^{\sharp}[E]$. Note first the important property connected with the Cartesian product. Let us start with the simplest case. We need new notation. For nonempty families $\mathcal{X}$ and $\mathcal{Y}$, we set

$$
\mathcal{X}\{\times\} \mathcal{Y} \triangleq\left\{\operatorname{pr}_{1}(z) \times \operatorname{pr}_{2}(z): z \in \mathcal{X} \times \mathcal{Y}\right\} .
$$

Family (6.1) is nonempty. We can use $\pi$-systems of nonempty sets as $\mathcal{X}$ and $\mathcal{Y}$. In addition, for all nonempty sets $X$ and $Y$ such that $\mathcal{X} \in \pi[X]$ and $\mathcal{Y} \in \pi[Y]$, we have

$$
\mathcal{X}\{\times\} \mathcal{Y} \in \pi[X \times Y] .
$$

Proposition 2. If $X$ and $Y$ are nonempty sets, $\mathcal{X} \in \pi_{\star}^{\sharp}[X]$ and $\mathcal{Y} \in \pi_{\star}^{\sharp}[Y]$, then

$$
\mathcal{X}\{\times\} \mathcal{Y} \in \pi_{\star}^{\sharp}[X \times Y] .
$$

P r o o f. Fix $X, Y, \mathcal{X}$, and $\mathcal{Y}$ satisfying our conditions. We have (6.2). Consider arbitrary sets

$$
\left(\Lambda_{1} \in \mathcal{X}\{\times\} \mathcal{Y}\right) \&\left(\Lambda_{2} \in \mathcal{X}\{\times\} \mathcal{Y}\right) \&\left(\Lambda_{3} \in \mathcal{X}\{\times\} \mathcal{Y}\right)
$$

Then, for some sets $U_{1} \in \mathcal{X}, V_{1} \in \mathcal{Y}, U_{2} \in \mathcal{X}, V_{2} \in \mathcal{Y}, U_{3} \in \mathcal{X}$, and $V_{3} \in \mathcal{Y}$, the following representation is valid:

$$
\left(\Lambda_{1}=U_{1} \times V_{1}\right) \&\left(\Lambda_{2}=U_{2} \times V_{2}\right) \&\left(\Lambda_{3}=U_{3} \times V_{3}\right) .
$$

Let relations (6.4) satisfy the conditions

$$
\left(\Lambda_{1} \cap \Lambda_{2} \neq \varnothing\right) \&\left(\Lambda_{2} \cap \Lambda_{3} \neq \varnothing\right) \&\left(\Lambda_{1} \cap \Lambda_{3} \neq \varnothing\right) .
$$

From (6.5) and (6.6), we obtain the following obvious properties:

$$
\left(U_{1} \cap U_{2} \neq \varnothing\right) \&\left(V_{1} \cap V_{2} \neq \varnothing\right) \&\left(U_{2} \cap U_{3} \neq \varnothing\right) \&\left(V_{2} \cap V_{3} \neq \varnothing\right) \&\left(U_{1} \cap U_{3} \neq \varnothing\right) \&\left(V_{1} \cap V_{3} \neq \varnothing\right) .
$$

From (1.3) and (6.7), we obtain

$$
\left(U_{1} \cap U_{2} \cap U_{3} \neq \varnothing\right) \&\left(V_{1} \cap V_{2} \cap V_{3} \neq \varnothing\right) .
$$

From (6.5) and (6.8), we obtain $\Lambda_{1} \cap \Lambda_{2} \cap \Lambda_{3} \neq \varnothing$. Thus (see (6.6)), the following implication hols:

$$
\left(\left(\Lambda_{1} \cap \Lambda_{2} \neq \varnothing\right) \&\left(\Lambda_{2} \cap \Lambda_{3} \neq \varnothing\right) \&\left(\Lambda_{1} \cap \Lambda_{3} \neq \varnothing\right)\right) \Longrightarrow\left(\Lambda_{1} \cap \Lambda_{2} \cap \Lambda_{3} \neq \varnothing\right) .
$$

Since the choice of (6.4) was arbitrary, we have proved (6.3) (see (1.3) and (6.2)).

We now return to the fixed nonempty set $E$ and suppose, unless otherwise stated, that $\mathcal{E} \in \pi_{\star}^{\sharp}[E]$ (see Proposition 1). In view of Proposition 1, we conclude that

$$
\left(\mathbb{F}_{0}^{\star}(\mathcal{E}), \mathbf{T}_{\mathcal{E}}^{0}\langle E\rangle\right)
$$

is a supercompact $T_{1}$-space (see [12, Sect. 5]). Recall Remark 1: in our case,

$$
\langle\mathcal{E}-\operatorname{link}\rangle_{0}[E]=\mathbb{F}_{0}^{\star}(\mathcal{E}) .
$$

From (3.8) and (6.10), we obtain the following equality for the topologies:

$$
\mathbf{T}_{\mathcal{E}}^{0}\langle E\rangle=\mathbb{T}_{0}\langle E \mid \mathcal{E}\rangle
$$

(we recall that, in our constructions, $\mathcal{E} \in \pi_{\star}^{\sharp}[E]$ ). Moreover, from (6.10), we obtain

$$
\langle\mathcal{E}-\operatorname{link}\rangle_{\mathrm{op}}^{0}[E \mid H]=\mathbb{F}_{\mathbf{C}}^{\natural}[\mathcal{E} \mid H] \quad \forall H \in \mathcal{P}(E) .
$$

As a corollary, we obtain the equality

$$
\hat{\mathfrak{C}}_{\mathrm{op}}^{0}[E ; \mathcal{E}]=\mathfrak{F}_{\mathbf{C}}^{\natural}[\mathcal{E}] .
$$


Proposition 3. If $\mathcal{G} \in \mathcal{P}^{\prime}\left(\mathbf{C}_{E}[\mathcal{E}]\right)$, then

$$
\left(\mathbb{F}_{0}^{\star}(\mathcal{E})=\bigcup_{\mathbb{G} \in \mathcal{G}} \mathbb{F}_{\mathbf{C}}^{\natural}[\mathcal{E} \mid \mathbb{G}]\right) \Longrightarrow\left(\exists G_{1} \in \mathcal{G} \exists G_{2} \in \mathcal{G}: \mathbb{F}_{0}^{\star}(\mathcal{L})=\mathbb{F}_{\mathbf{C}}^{\natural}\left[\mathcal{E} \mid G_{1}\right] \cup \mathbb{F}_{\mathbf{C}}^{\natural}\left[\mathcal{E} \mid G_{2}\right]\right) .
$$

The corresponding proof follows from $(6.10),(6.12)$, and the properties of the family $\hat{\mathfrak{C}}_{\mathrm{op}}^{0}[E ; \mathcal{E}]$ mentioned in [12, Sect. 5]. In (6.13), we obtained a natural sharpening of Proposition 1.

Remark 2. Note that, by duality, the family $(\mathbb{U F})[E ; \mathcal{E}]$ in the considered case is a closed binary subbase (see $[12,(5.1)]$ ) of the topological space (6.9). This property follows from [12, Proposition 5.1] and (6.12).

In the next section, we consider specific examples, for which (6.10) and (6.11) are realized.

\section{Examples of supercompact ultrafilter spaces}

We first consider the cases when $E \subset \mathbb{R}$, where $\mathbb{R}$ is the real line (Proposition 2 enables extending our constructions to a vector case). Consider the case when this set $E$ is bounded. More precisely, suppose that $E$ is an interval of $\mathbb{R}$. In addition, we will consider specific sets $E$.

Let us agree to use only square brackets in the notation of all intervals in $\mathbb{R}:] x, y[,[x, y[] x, y$,$] ,$ and $[x, y]$ for $x \in \mathbb{R}$ and $y \in \mathbb{R}$. In addition, relations between $x$ and $y$ can be arbitrary: $x<y, x=y$, and $y<x$. Thus, for example, $[1,0] \triangleq\{\xi \in \mathbb{R} \mid(1 \leq \xi) \&(\xi \leq 0)\}=\varnothing$. In other words, we consider the empty set as an interval. Moreover, for $c \in \mathbb{R}$, we set (as usually) $]-\infty, c[\triangleq\{\xi \in \mathbb{R} \mid \xi<c\}$ and ]$c, \infty[\triangleq\{\xi \in \mathbb{R} \mid c<\xi\}$.

For $a \in \mathbb{R}$ and $b \in] a, \infty[$, define

$$
\begin{aligned}
& \mathcal{J}_{1}(a, b) \triangleq\left\{\operatorname{pr}_{1}(z), \operatorname{pr}_{2}(z)[: z \in[a, b] \times[a, b]\},\right. \\
& \mathcal{J}_{2}(a, b) \triangleq\left\{\left[\operatorname{pr}_{1}(z), \operatorname{pr}_{2}(z)\right]: z \in[a, b] \times[a, b]\right\}, \\
& \mathcal{J}_{3}(a, b) \triangleq\left\{\left[\operatorname{pr}_{1}(z), \operatorname{pr}_{2}(z)[: z \in[a, b] \times[a, b]\},\right.\right. \\
&\left.\mathcal{J}_{4}(a, b) \triangleq\left\{\operatorname{pr}_{1}(z), \operatorname{pr}_{2}(z)\right]: z \in[a, b] \times[a, b]\right\} .
\end{aligned}
$$

We obtain four nonempty families of subsets of $\mathbb{R}$.

Proposition 4. If $a \in \mathbb{R}$ and $b \in] a, \infty[$, then

$$
\left(\mathcal{J}_{1}(a, b) \in \pi_{\star}^{\sharp}[] a, b[]\right) \&\left(\mathcal{J}_{2}(a, b) \in \pi_{\star}^{\sharp}[[a, b]]\right) \&\left(\mathcal{J}_{3}(a, b) \in \pi_{\star}^{\sharp}\left[[a, b[]) \&\left(\mathcal{J}_{4}(a, b) \in \pi_{\star}^{\sharp}[] a, b\right]\right]\right) .
$$

P r o o f. The proofs of all four statements in (7.1) are similar. Therefore, let us consider only one of them and establish the property $\mathcal{J}_{1}(a, b) \in \pi_{\star}^{\sharp}[] a, b[]$, where $a \in \mathbb{R}, b \in \mathbb{R}$, and $a<b$. Note the obvious property:

$$
\mathcal{J}_{1}(a, b) \in \pi[] a, b[] \text {. }
$$

Let $\Lambda_{1} \in \mathcal{J}_{1}(a, b), \Lambda_{2} \in \mathcal{J}_{1}(a, b)$, and $\Lambda_{3} \in \mathcal{J}_{1}(a, b)$. Then, for some numbers $u_{1} \in[a, b], u_{2} \in[a, b]$, $v_{1} \in[a, b], v_{2} \in[a, b], w_{1} \in[a, b]$, and $w_{2} \in[a, b]$, we have

$$
\left(\Lambda_{1}=\right] u_{1}, u_{2}[) \&\left(\Lambda_{2}=\right] v_{1}, v_{2}[) \&\left(\Lambda_{3}=\right] w_{1}, w_{2}[) .
$$

Let $\left(\Lambda_{1} \cap \Lambda_{2} \neq \varnothing\right) \&\left(\Lambda_{2} \cap \Lambda_{3} \neq \varnothing\right) \&\left(\Lambda_{1} \cap \Lambda_{3} \neq \varnothing\right)$. We introduce $\mu \triangleq \sup \left(\left\{u_{1} ; v_{1} ; w_{1}\right\}\right) \in[a, b]$ and $\nu \triangleq \inf \left(\left\{u_{2} ; v_{2} ; w_{2}\right\}\right) \in[a, b]$. Then, by (7.3), ] $\mu, \nu\left[\subset \Lambda_{1} \cap \Lambda_{2} \cap \Lambda_{3}\right.$ and $\mu<\nu$ (the latter inequality 
follows from the linkedness of the family $\left\{\Lambda_{1} ; \Lambda_{2} ; \Lambda_{3}\right\}$ ). As a result, $\Lambda_{1} \cap \Lambda_{2} \cap \Lambda_{3} \neq \varnothing$. Thus, we obtain the implication

$$
\left(\left(\Lambda_{1} \cap \Lambda_{2} \neq \varnothing\right) \&\left(\Lambda_{2} \cap \Lambda_{3} \neq \varnothing\right) \&\left(\Lambda_{1} \cap \Lambda_{3} \neq \varnothing\right)\right) \Longrightarrow\left(\Lambda_{1} \cap \Lambda_{2} \cap \Lambda_{3} \neq \varnothing\right) .
$$

Since $\Lambda_{1}, \Lambda_{2}$, and $\Lambda_{3}$ were chosen arbitrary, the required property of the family $\mathcal{J}_{1}(a, b)$ follows from (1.3), (7.2), and (7.4).

Thus, by Propositions 2 and 4, we obtain many examples of realization of (6.10) and (6.11). In these examples, (6.9) is a supercompact $T_{1}$-space (see Proposition 1 ). Later, we will provide analogs of (7.1) for the case $E=\mathbb{R}$. Now we first observe a very simple general construction.

Unless otherwise stated, we suppose that $E$ is an arbitrary nonempty set. Suppose that

$$
\pi_{E}^{0} \triangleq\left\{\mathcal{L} \in \mathcal{P}^{\prime}(\mathcal{P}(E)) \mid(\varnothing \in \mathcal{L}) \&(A \cap B \in \mathcal{L} \quad \forall A \in \mathcal{L} \quad \forall B \in \mathcal{L})\right\}
$$

Certainly, $\pi[E]=\left\{\mathcal{L} \in \pi_{E}^{0} \mid E \in \mathcal{L}\right\}$. It is obvious that $\mathcal{L} \cup\{E\} \in \pi[E] \quad \forall \mathcal{L} \in \pi_{E}^{0}$. Moreover, let

$$
\begin{gathered}
\pi_{E}^{\sharp} \triangleq\left\{\mathcal{L} \in \pi_{E}^{0} \mid \forall L_{1} \in \mathcal{L} \quad \forall L_{2} \in \mathcal{L} \quad \forall L_{3} \in \mathcal{L}\right. \\
\left.\left(\left(L_{1} \cap L_{2} \neq \varnothing\right) \&\left(L_{2} \cap L_{3} \neq \varnothing\right) \&\left(L_{1} \cap L_{3} \neq \varnothing\right)\right) \Longrightarrow\left(L_{1} \cap L_{2} \cap L_{3} \neq \varnothing\right)\right\} .
\end{gathered}
$$

Then, we obtain the following obvious property:

$$
\mathcal{L} \cup\{E\} \in \pi_{\star}^{\sharp}[E] \quad \forall \mathcal{L} \in \pi_{E}^{\sharp} .
$$

In (7.5)-(7.7), we have simple modifications of definitions of Section 1 (see (1.2) and (1.3)). Consider now the case when $E=\mathbb{R}$. In addition,

$$
\begin{aligned}
& \left(\mathcal{J}_{\mathbb{R}}^{(1)} \triangleq\{]_{p_{1}}(z), \operatorname{pr}_{2}(z)[: \quad z \in \mathbb{R} \times \mathbb{R}\} \in \pi_{\mathbb{R}}^{\sharp}\right) \\
& \&\left(\mathcal{J}_{\mathbb{R}}^{(2)} \triangleq\left\{\left[\operatorname{pr}_{1}(z), \operatorname{pr}_{2}(z)\right]: z \in \mathbb{R} \times \mathbb{R}\right\} \in \pi_{\mathbb{R}}^{\sharp}\right) \\
& \&\left(\mathcal { J } _ { \mathbb { R } } ^ { ( 3 ) } \triangleq \left\{\left[\operatorname{pr}_{1}(z), \operatorname{pr}_{2}(z)[: z \in \mathbb{R} \times \mathbb{R}\} \in \pi_{\mathbb{R}}^{\sharp}\right)\right.\right. \\
& \left.\&\left(\mathcal{J}_{\mathbb{R}}^{(4)} \triangleq\left\{\operatorname{lpr}_{1}(z), \operatorname{pr}_{2}(z)\right]: z \in \mathbb{R} \times \mathbb{R}\right\} \in \pi_{\mathbb{R}}^{\sharp}\right) .
\end{aligned}
$$

The corresponding proof is an obvious analog of constructions used in the proof of Proposition 4 . By (7.8), we obtain new variants of (6.10), (6.11), and Proposition 1 for the case when $E=\mathbb{R}$ and

$$
\left(\mathcal{E}=\mathcal{J}_{\mathbb{R}}^{(1)} \cup\{\mathbb{R}\}\right) \vee\left(\mathcal{E}=\mathcal{J}_{\mathbb{R}}^{(2)} \cup\{\mathbb{R}\}\right) \vee\left(\mathcal{E}=\mathcal{J}_{\mathbb{R}}^{(3)} \cup\{\mathbb{R}\}\right) \vee\left(\mathcal{E}=\mathcal{J}_{\mathbb{R}}^{(4)} \cup\{\mathbb{R}\}\right) .
$$

The direct combination of Propositions 2 and 4 and similar combination of Proposition 2 and (7.8) enable obtaining variants of (6.10), (6.11), and Proposition 1 for subsets of $\mathbb{R} \times \mathbb{R}$.

\section{The case of generalized Cartesian product}

In this section, we consider a natural generalization of Proposition 2. More precisely, we fix nonempty sets $X$ and $E$. In what follows, for every nonempty set $S$, we denote by $S^{X}$ (as in [17, Ch. II, Sect. 6]) the set of all mappings from $X$ to $S$; we can use a nonempty family as $S$. In addition, we fix a (set-valued) mapping $\left(E_{x}\right)_{x \in X} \in \mathcal{P}^{\prime}(E)^{X}$; thus, we have a mapping

$$
x \longmapsto E_{x}: X \longrightarrow \mathcal{P}^{\prime}(E) .
$$


Using (8.1) (and the axiom of choice), we obtain the (nonempty) set

$$
\mathbb{E} \triangleq \prod_{x \in X} E_{x}=\left\{f \in E^{X} \mid f(x) \in E_{x} \quad \forall x \in X\right\} \in \mathcal{P}^{\prime}\left(E^{X}\right) .
$$

In connection with (8.1) and (8.2), note that

$$
\prod_{x \in X} \pi\left[E_{x}\right]=\left\{\left(\mathcal{L}_{x}\right)_{x \in X} \in \mathcal{P}^{\prime}(\mathcal{P}(E))^{X} \mid \mathcal{L}_{y} \in \pi\left[E_{y}\right] \quad \forall y \in X\right\}
$$

Similarly, we define the set

$$
\prod_{x \in X} \pi_{\star}^{\sharp}\left[E_{x}\right]=\left\{\left(\mathcal{L}_{x}\right)_{x \in X} \in \mathcal{P}^{\prime}(\mathcal{P}(E))^{X} \mid \mathcal{L}_{y} \in \pi_{\star}^{\sharp}\left[E_{y}\right] \quad \forall y \in X\right\} \in \mathcal{P}\left(\prod_{x \in X} \pi\left[E_{x}\right]\right) .
$$

From (8.3), we obtain the following obvious representation:

$$
\prod_{x \in X} \mathcal{L}_{x}=\left\{\left(L_{x}\right)_{x \in X} \in \mathcal{P}(E)^{X} \mid L_{y} \in \mathcal{L}_{y} \quad \forall y \in X\right\} \in \mathcal{P}^{\prime}\left(\mathcal{P}(E)^{X}\right) \quad \forall\left(\mathcal{L}_{x}\right)_{x \in X} \in \prod_{x \in X} \pi\left[E_{x}\right] .
$$

As a corollary, by (8.4), the following product of sets is defined:

$$
\prod_{x \in X} L_{x}=\left\{f \in E^{X} \mid f(x) \in L_{x} \forall x \in X\right\} \quad \forall\left(\mathcal{L}_{x}\right)_{x \in X} \in \prod_{x \in X} \pi\left[E_{x}\right] \quad \forall\left(L_{x}\right)_{x \in X} \in \prod_{x \in X} \mathcal{L}_{x} .
$$

Now, we introduce two variants of the $\pi$-system product: usual (similar to the variant applied in the general topology in the Tychonoff product construction) and "box" variants. The latter variant corresponds to the way used in the construction of the box topology.

More precisely (see (8.4) and (8.5)), we introduce the usual $\pi$-system product for $\left(\mathcal{L}_{x}\right)_{x \in X} \in \prod_{x \in X} \pi\left[E_{x}\right]$ :

$$
\begin{gathered}
\bigotimes_{x \in X} \mathcal{L}_{x} \triangleq\left\{H \in \mathcal{P}\left(E^{X}\right) \mid \exists\left(L_{x}\right)_{x \in X} \in \prod_{x \in X} \mathcal{L}_{x}:\left(H=\prod_{x \in X} L_{x}\right) \&(\exists K \in \operatorname{Fin}(X):\right. \\
\left.\left.L_{s}=E_{s} \quad \forall s \in X \backslash K\right)\right\}=\left\{H \in \mathcal{P}(\mathbb{E}) \mid \exists\left(L_{x}\right)_{x \in X} \in \prod_{x \in X} \mathcal{L}_{x}:\right. \\
\left.\left(H=\prod_{x \in X} L_{x}\right) \&\left(\exists K \in \operatorname{Fin}(X): L_{s}=E_{s} \quad \forall s \in X \backslash K\right)\right\} .
\end{gathered}
$$

The "box" variant is defined easier. For $\left(\mathcal{L}_{x}\right)_{x \in X} \in \prod_{x \in X} \pi\left[E_{x}\right]$, we set

$$
\bigodot_{x \in X} \mathcal{L}_{x} \triangleq\left\{\prod_{x \in X} L_{x}:\left(L_{x}\right)_{x \in X} \in \prod_{x \in X} \mathcal{L}_{x}\right\}
$$

We note two obvious (but useful) properties. More precisely, for $\left(H_{x}^{(1)}\right)_{x \in X} \in \mathcal{P}(E)^{X}$ and $\left(H_{x}^{(2)}\right)_{x \in X} \in \mathcal{P}(E)^{X}$, we have

$$
\left(\prod_{x \in X} H_{x}^{(1)}\right) \cap\left(\prod_{x \in X} H_{x}^{(2)}\right)=\prod_{x \in X}\left(H_{x}^{(1)} \cap H_{x}^{(2)}\right) .
$$

Similarly, for $\left(H_{x}^{(1)}\right)_{x \in X} \in \mathcal{P}(E)^{X},\left(H_{x}^{(2)}\right)_{x \in X} \in \mathcal{P}(E)^{X}$, and $\left(H_{x}^{(3)}\right)_{x \in X} \in \mathcal{P}(E)^{X}$, we have

$$
\left(\prod_{x \in X} H_{x}^{(1)}\right) \cap\left(\prod_{x \in X} H_{x}^{(2)}\right) \cap\left(\prod_{x \in X} H_{x}^{(3)}\right)=\prod_{x \in X}\left(H_{x}^{(1)} \cap H_{x}^{(2)} \cap H_{x}^{(3)}\right) .
$$


From (8.6) and (8.8), we obtain

$$
\bigotimes_{x \in X} \mathcal{L}_{x} \in \pi[\mathbb{E}] \quad \forall\left(\mathcal{L}_{x}\right)_{x \in X} \in \prod_{x \in X} \pi\left[E_{x}\right]
$$

Moreover, (1.2), (8.7), and (8.8) imply

$$
\bigodot_{x \in X} \mathcal{L}_{x} \in \pi[\mathbb{E}] \quad \forall\left(\mathcal{L}_{x}\right)_{x \in X} \in \prod_{x \in X} \pi\left[E_{x}\right]
$$

In addition, we obtain the following obvious inclusion for $\left(\mathcal{L}_{x}\right)_{x \in X} \in \prod_{x \in X} \pi\left[E_{x}\right]$ :

$$
\bigotimes_{x \in X} \mathcal{L}_{x} \subset \bigodot_{x \in X} \mathcal{L}_{x}
$$

Theorem 2. If $\left(\mathcal{L}_{x}\right)_{x \in X} \in \prod_{x \in X} \pi_{\star}^{\sharp}\left[E_{x}\right]$, then

$$
\left(\bigotimes_{x \in X} \mathcal{L}_{x} \in \pi_{\star}^{\sharp}[\mathbb{E}]\right) \&\left(\bigodot_{x \in X} \mathcal{L}_{x} \in \pi_{\star}^{\sharp}[\mathbb{E}]\right) .
$$

P r o o f. We restrict ourselves to the verification of the latter inclusion in (8.12) (the corresponding verification of the former inclusion is an obvious corollary; see (8.11)). In this proof, we fix

$$
\left(\mathcal{L}_{x}\right)_{x \in X} \in \prod_{x \in X} \pi_{\star}^{\sharp}\left[E_{x}\right]
$$

and use (8.10). From (8.10) and (8.13), we obtain

$$
\left(\mathcal{L}_{x}\right)_{x \in X} \in \prod_{x \in X} \pi\left[E_{x}\right]: \mathfrak{L} \triangleq \bigodot_{x \in X} \mathcal{L}_{x} \in \pi[\mathbb{E}] .
$$

Thus, by (8.7) and (8.14), we have the following equality:

$$
\mathfrak{L}=\left\{\prod_{x \in X} L_{x}:\left(L_{x}\right)_{x \in X} \in \prod_{x \in X} \mathcal{L}_{x}\right\} .
$$

Consider arbitrary sets $\Lambda_{1} \in \mathfrak{L}, \Lambda_{2} \in \mathfrak{L}$, and $\Lambda_{3} \in \mathfrak{L}$. Taking into account (8.15), we choose

$$
\left(\left(U_{x}\right)_{x \in X} \in \prod_{x \in X} \mathcal{L}_{x}\right) \&\left(\left(V_{x}\right)_{x \in X} \in \prod_{x \in X} \mathcal{L}_{x}\right) \&\left(\left(W_{x}\right)_{x \in X} \in \prod_{x \in X} \mathcal{L}_{x}\right)
$$

with the following properties:

$$
\left(\Lambda_{1}=\prod_{x \in X} U_{x}\right) \&\left(\Lambda_{2}=\prod_{x \in X} V_{x}\right) \&\left(\Lambda_{3}=\prod_{x \in X} W_{x}\right) .
$$

Then, by (8.8) and (8.16), we conclude that $\Lambda_{1} \cap \Lambda_{2}, \Lambda_{2} \cap \Lambda_{3}$, and $\Lambda_{1} \cap \Lambda_{3}$ are realized as follows:

$$
\left(\Lambda_{1} \cap \Lambda_{2}=\prod_{x \in X}\left(U_{x} \cap V_{x}\right)\right) \&\left(\Lambda_{2} \cap \Lambda_{3}=\prod_{x \in X}\left(V_{x} \cap W_{x}\right)\right) \&\left(\Lambda_{1} \cap \Lambda_{3}=\prod_{x \in X}\left(U_{x} \cap W_{x}\right)\right) .
$$

Similarly, from (8.9) and (8.16), we obtain

$$
\Lambda_{1} \cap \Lambda_{2} \cap \Lambda_{3}=\prod_{x \in X}\left(U_{x} \cap V_{x} \cap W_{x}\right) .
$$


Suppose now that

$$
\left(\Lambda_{1} \cap \Lambda_{2} \neq \varnothing\right) \&\left(\Lambda_{2} \cap \Lambda_{3} \neq \varnothing\right) \&\left(\Lambda_{1} \cap \Lambda_{3} \neq \varnothing\right) .
$$

Then, from (8.17) and (8.19), we obtain, for $x \in X$,

$$
\left(U_{x} \cap V_{x} \neq \varnothing\right) \&\left(V_{x} \cap W_{x} \neq \varnothing\right) \&\left(U_{x} \cap W_{x} \neq \varnothing\right),
$$

where $U_{x} \in \mathcal{L}_{x}, V_{x} \in \mathcal{L}_{x}$, and $W_{x} \in \mathcal{L}_{x}$. However, for $x \in X$, the inclusion $\mathcal{L}_{x} \in \pi_{\star}^{\sharp}\left[E_{x}\right]$ holds; therefore, by (1.3) and (8.20), we have $U_{x} \cap V_{x} \cap W_{x} \neq \varnothing$. Using (8.18) (and the axiom of choice), we obtain $\Lambda_{1} \cap \Lambda_{2} \cap \Lambda_{3} \neq \varnothing$. Thus, we have proved the implication

$$
\left(\left(\Lambda_{1} \cap \Lambda_{2} \neq \varnothing\right) \&\left(\Lambda_{2} \cap \Lambda_{3} \neq \varnothing\right) \&\left(\Lambda_{1} \cap \Lambda_{3} \neq \varnothing\right)\right) \Longrightarrow\left(\Lambda_{1} \cap \Lambda_{2} \cap \Lambda_{3} \neq \varnothing\right) .
$$

Since $\Lambda_{1}, \Lambda_{2}$, and $\Lambda_{3}$ were chosen arbitrary, by (8.21), we obtain (see (1.3)) $\mathfrak{L} \in \pi_{\star}^{\sharp}[\mathbb{E}]$. By (8.14), we have

$$
\bigodot_{x \in X} \mathcal{L}_{x} \in \pi_{\star}^{\sharp}[\mathbb{E}] .
$$

Thus, the theorem is proved (see (1.3) and (8.11)).

Now, we observe some cases connected with the realization of our measurable space equipped with the semi-algebra of sets. These measurable spaces are widely used in the probability theory (see [1]). Let us first give a general definition in terms of $\pi$-systems. For this, we fix a nonempty set $\mathbb{X}$. If $\mathcal{L} \in \pi[\mathbb{X}], A \in \mathcal{P}(\mathbb{X})$, and $n \in \mathbb{N}$, then

$$
\Delta_{n}(A, \mathcal{L}) \triangleq\left\{\left(L_{i}\right)_{i \in \overline{1, n}} \in \mathcal{L}^{n} \mid\left(A=\bigcup_{i=1}^{n} L_{i}\right) \&\left(L_{p} \cap L_{q}=\varnothing \quad \forall p \in \overline{1, n} \quad \forall q \in \overline{1, n} \backslash\{p\}\right)\right\} .
$$

We set

$$
\Pi[\mathbb{X}] \triangleq\left\{\mathcal{L} \in \pi[\mathbb{X}] \mid \forall L \in \mathcal{L} \quad \exists n \in \mathbb{N}: \Delta_{n}(\mathbb{X} \backslash L, \mathcal{L}) \neq \varnothing\right\}
$$

thus, the family of all semi-algebras of subsets of $\mathbb{X}$ is introduced. Certainly, $(\operatorname{alg})[\mathbb{X}] \subset \Pi[\mathbb{X}]$. Moreover,

$$
\begin{gathered}
\Pi_{\star}^{\sharp}[\mathbb{X}] \triangleq\left\{\mathcal{L} \in \Pi[\mathbb{X}] \mid \forall L_{1} \in \mathcal{L} \forall L_{2} \in \mathcal{L} \forall L_{3} \in \mathcal{L}\right. \\
\left.\left(\left(L_{1} \cap L_{2} \neq \varnothing\right) \&\left(L_{2} \cap L_{3} \neq \varnothing\right) \&\left(L_{1} \cap L_{3} \neq \varnothing\right)\right) \Longrightarrow\left(L_{1} \cap L_{2} \cap L_{3} \neq \varnothing\right)\right\} \\
=\Pi[\mathbb{X}] \cap \pi_{\star}^{\sharp}[\mathbb{X}] .
\end{gathered}
$$

Elements of family (8.22) are special semi-algebras of subsets of $\mathbb{X}$. Note that, for $a \in \mathbb{R}$ and $b \in] a, \infty\left[\right.$, by Proposition $4, \mathcal{J}_{3}(a, b) \in \Pi_{*}^{\sharp}[[a, b[]$ and

$$
\left(\left[a, b\left[, \mathcal{J}_{3}(a, b)\right)\right.\right.
$$

is the usual space-pointer. Of course, $(8.23)$ is a specific measurable space with a semi-algebra of sets. In addition, by Proposition $4, \mathcal{J}_{3}(a, b) \in \pi_{\star}^{\sharp}\left[\left[a, b[]\right.\right.$. Thus, for $E=\left[a, b\left[\right.\right.$ and $\mathcal{E}=\mathcal{J}_{3}(a, b)$, we obtain (6.10) and (6.11).

Consider a more complicated case. We return to (8.1) and (8.2). For a nonempty set $X$ and $\left(E_{x}\right)_{x \in X} \in \mathcal{P}^{\prime}(E)^{X}$, we introduce

$$
\prod_{x \in X} \Pi\left[E_{x}\right] \triangleq\left\{\left(\mathcal{L}_{x}\right)_{x \in X} \in \mathcal{P}^{\prime}(\mathcal{P}(E))^{X} \mid \mathcal{L}_{y} \in \Pi\left[E_{y}\right] \forall y \in X\right\} \in \mathcal{P}\left(\prod_{x \in X} \pi\left[E_{x}\right]\right)
$$


By (8.14) and (8.24), we define

$$
\left.\bigotimes_{x \in X} \mathcal{L}_{x} \in \pi[\mathbb{E}] \forall \mathcal{L}_{x}\right)_{x \in X} \in \prod_{x \in X} \Pi\left[E_{x}\right]
$$

Moreover, the following property holds:

$$
\left.\bigotimes_{x \in X} \mathcal{L}_{x} \in \Pi[\mathbb{E}] \forall \mathcal{L}_{x}\right)_{x \in X} \in \prod_{x \in X} \Pi\left[E_{x}\right] .
$$

From Theorem 2 and (8.25), we obtain for $\left(\mathcal{L}_{x}\right)_{x \in X} \in \prod_{x \in X} \Pi_{\star}^{\sharp}\left[E_{x}\right]$

$$
\bigotimes_{x \in X} \mathcal{L}_{x} \in \Pi_{\star}^{\sharp}[\mathbb{E}] .
$$

Note an obvious corollary concerning the case $E=\mathbb{R}$.

Proposition 5. Let $\left(a_{x}\right)_{x \in X} \in \mathbb{R}^{X}$ and $\left.\left(b_{x}\right)_{x \in X} \in \prod_{x \in X}\right] a_{x}, \infty[$. Then

$$
\bigotimes_{x \in X} \mathcal{J}_{3}\left(a_{x}, b_{x}\right) \in \Pi_{\star}^{\sharp}\left[\left\{f \in \mathbb{R}^{X} \mid \forall x \in X \quad\left(a_{x} \leq f(x)\right) \&\left(f(x)<b_{x}\right)\right\}\right] .
$$

The corresponding proof is obtained by the direct combination of (8.26) and the abovementioned properties of the measurable space (8.23). Thus, by (8.22) and (8.25), we obtain one more variant of realization of (6.10) and (6.11) in the class of measurable spaces with a semi-algebra of sets (see Proposition 1).

\section{Conclusion}

In this paper, relations between ultrafilters and maximal linked systems on a $\pi$-system were considered. The emphasis was on the conditions under which all maximal linked systems are ultrafilters on the $\pi$-system. The investigation of the ultrafilter space is important, since in the class of ultrafilters the density properties (see (4.5) and (4.6)) are realized. Therefore, this space can be considered as a kind of extension of the initial set $E$ whose elements play the role of usual solutions. Such interpretation is very natural for extension procedures of extremal problems and attainability problems (see $[2,3,19,20]$ ). More precisely, the ultrafilters can be used as generalized elements. Therefore, it is useful to know as much as possible properties of topological nature. The supercompactness is a very interesting property of such type. We investigate this property identifying ultrafilters and maximal linked systems for a $\pi$-system. Of course, this approach is not universal. However, we obtain a positive result for many interesting variants of $\pi$-systems. In addition, we use the supercompactness property of the space of maximal linked systems with Wallman equipping.

\section{REFERENCES}

1. Bulinskii A.V., Shiryaev A. N. Teoriya sluchainykh processov [Theory of Random Processes]. Moscow: Fizmatlit, 2005. 402 p. (in Russian)

2. Chentsov A. G. One representation of the results of action of approximate solutions in a problem with constraints of asymptotic nature. Proc. Steklov Inst. Math., 2012. Vol. 276. Suppl. 1. P. S48-S62. DOI: $10.1134 / \mathrm{S} 0081543812020046$ 
3. Chentsov A. G. On example of ultrafilter space of algebra of sets. Tr. Inst. Mat. Mekh. UrO RAN, 2011. Vol. 17, No. 4. P. 293-311. (in Russian)

4. Arhangel'skii A. V. Compactness. Itogi Nauki i Tekhniki. Ser. Sovrem. Probl. Mat. Fund. Napr., VINITI, 1989. Vol. 50. P. 5-128. (in Russian)

5. Engelking R. General Topology. Warsaw: PWN, 1977. 751 p.

6. De Groot J. Superextensions and supercompactness. "Contributions to Extension Theory of Topological Structures and Its Applications": Proc. Int. Symp. (Germany, Berlin, August 14-19, 1967). Berlin: Deutscher Verlag Wiss., 1969. P. 89-90.

7. Van Mill J. Supercompactness and Wallman Spaces. Amsterdam: Math. Centre Tracts, vol. 85, 1977. $238 \mathrm{p}$.

8. Strok M., Szymański A. Compact metric spaces have binary bases. Fund. Math., 1975. Vol. 89. P. 81-91. DOI: $10.4064 / \mathrm{fm}-89-1-81-91$

9. Fedorchuk V.V., Filippov V. V. Obshchaya topologiya. Osnovnye konstrukcii [General Topology. Basic Constructions]. Moscow: Fizmatlit, 2006. 332 p. (in Russian)

10. Chentsov A. G. Superextension as a bitopological space. Izv. Inst. Mat. Informat. Udmurt. Univ., 2017. Vol. 49. P. 55-79. (in Russian) DOI: 10.20537/2226-3594-2017-49-03

11. Chentsov A. G. Ultrafilters and maximal linked systems of sets. Vestn. Udmurt. Univ. Mat. Mekh. Komp. Nauki, 2017. Vol. 27, No. 3. P. 365-388. (in Russian) DOI: 10.20537/vm170307

12. Chentsov A. G. Bitopological spaces of ultrafilters and maximal linked systems. Tr. Inst. Mat. Mekh. UrO RAN, 2018. Vol. 24, No. 1. P. 257-272. (in Russian) DOI: 10.21538/0134-4889-2018-24-1-257-272

13. Chentsov A. G. Some representations connected with ultrafilters and maximal linked systems. Ural Math. J., 2017. Vol. 3, No. 2. P. 100-121. DOI: 10.15826/umj.2017.2.012

14. Chentsov A. G. To the question on abstract analogs of superextensions of topological spaces. Functionally Differential Equations: Theory and Applications: Proc. Conf. Perm: Perm Nat. Research Polytech. Univ., 2018. P. 244-262. (in Russian)

15. Chentsov A.G. Maximal linked systems and ultrafilters of widely understood mesurable spaces. Tambov Univ. Reports. Ser. Nat. Tech. Sci., 2018. Vol. 23, No. 124. P. 846-860. (in Russian) DOI: 10.20310/1810-0198-2018-23-124-846-860

16. Chentsov A.G. Ultrafilters and maximal linked systems: basic properies and topological constructions. Izv. Inst. Mat. Informat. Udmurt. Univ., 2018. Vol. 52. P. 86-102. (in Russian) DOI: $10.20537 / 2226-3594-2018-52-07$

17. Kuratowski K., Mostowski A. Set Theory. Amsterdam: North Holland Publishing Company, 1967. 416 p.

18. Aleksandrov P.S. Vvedenie v teoriyu mnozhestv $i$ obshchuyu topologiyu [Introduction to the Theory of Sets and General Topology]. Moscow: Editorial URSS, 2004. 366 p. (in Russian)

19. Chentsov A. G. Attraction sets in abstract attainability problems: equivalent representations and basic properties. Russian Math. (Iz. VUZ), 2013. Vol. 57, No. 11. P. 28-44. DOI: 10.3103/S1066369X13110030

20. Chentsov A. G. Ultrafilters of measurable spaces as generalized solutions in abstract attainability problem. Proc. Steklov Inst. Math., 2011. Vol. 275. Suppl. 1. P. S12-S39. DOI: 10.1134/S0081543811090021 